\title{
Free vibration of rectangular liquid tanks: experiments and the finite element method
}

\author{
A. S. Ghods ${ }^{1}$, M. R. Esfahani ${ }^{2}$ \& A. Aftabi Sani ${ }^{3}$ \\ ${ }^{1}$ Islamic Azad University, Mashhad Branch, Iran \\ ${ }^{2}$ Ferdowsi University of Mashhad, Iran \\ ${ }^{3}$ Islamic Azad University, Mashhad Branch, Iran
}

\begin{abstract}
The experimental study of structural vibration is often performed to determine the modal parameters of a structure or to verify the theoretical models and predictions. The first phase of this research involved the experimental determination of the modal properties of a rectangular steel tank with different levels of water. The natural frequencies obtained from the experiments were compared to those calculated by the analytical models. In the second phase, a finite element analysis is carried out. The numerical results are compared with the experimental values, and a very good agreement between experimental and numerical results was obtained.
\end{abstract}

Keywords: modal test, rectangular tank, fluid-structure interaction, finite element method.

\section{Introduction}

For several decades, the fluid-structure interaction problem has been a continuous challenging research subject in various scientific and engineering applications, such as stationary liquid storage tanks, dam-reservoir systems, nuclear reactors in fluid and tower-like structures (Rebouillat and Liksonov [1]). The dynamic interaction between an elastic structure and a compressible fluid has been the subject of intensive investigations in recent years. At present, the most common approach being adopted is that the fluid and structure are coupled and solved as one system. This system can be solved by numerous numerical methods, such as finite element (FEM) (Ghaemmaghami and Kianoush [2]), boundary element (BEM) (Kim et al. [3]) and coupled boundary element-finite 
element method (BEM/FEM) (Koh et al. [4]). These methods have provided many useful and satisfactory results.

Studies on the seismic response of rectangular tanks are not adequate, while those concerning cylindrical tanks are numerous. Moreover, in most existing studies on the rectangular tanks, the tank structures are assumed to be rigid. Kim et al. [3] studied the dynamic behavior of 3-D rectangular flexible fluid containers using the Rayleigh-Ritz method. Park et al. [5] studied the dynamic behavior of rectangular concrete tanks using boundary element modeling for the fluid motion and finite element modeling for the solid walls. The time-history analytical method was used to obtain the dynamic response of fluid storage tank subjected to earthquakes. Both impulsive and convective effects were considered. Later, they presented an analytical method using three-dimensional hydrodynamic pressure calculations. Chen and Kianoush [6] proposed a sequential method to determine the hydrodynamic pressures of rectangular tanks by considering the effect of wall flexibility on impulsive pressure.

Including the wall deformability in a dynamic analysis requires a systematic knowledge and understanding of the fluid-structure free-vibrational characteristics. Generally, research has been focused on the natural frequencies, while vibration mode shapes and modal damping have been overlooked (Virella et al. [7]). Over the last decade, a number of new studies on the dynamics of partial filled cylindrical shells have been published (Biswal et al. [8]).

One form of non-destructive testing that may be used to obtain information on the dynamic behavior of actual structures is the experimental modal analysis. The experimental study of structural vibration is often performed to determine the modal parameters of a structure (i.e. natural frequencies, damping and mode shapes), or to verify the theoretical models and predictions. This technique has the advantage of determining the real structural properties without using any assumption and taking into account the actual boundary condition.

In this paper, a numerical and experimental study is undertaken to provide a deeper understanding of the effect of different parameters on the modal characteristics and hydrodynamic pressures in steel rectangular tanks. The results of this study can be used as the base for further studies on the behavior of reinforced concrete rectangular tanks which are widely used in practical.

\section{Modal test description}

The structural testing setup includes elements for excitation, response, measurement and analysis. An impact hammer is the most convenient method of exciting a structure. It resembles an ordinary hammer but has a force transducer built into the tip to register the force input. The resulting response of the structure is registered by one accelerometer. The measuring process is carried out using a two-channel signal analyzer.

It transforms both the excitation and response time histories into frequency spectra and calculates the ratio of these two functions, referred to as the frequency response function (FRF). The FRF is a property of the linear dynamic system, independent of the type of the excitation function. 
In the dynamic test, the impact hammer provided the excitation input at every degree of freedom, and the frequency response function (FRF) was derived by measuring the input force and response at each measurement point using Eq. (1).

$$
F R F(f)=G_{y x}(f) / G_{x x}(f)
$$

Where $G_{y x}(f)$ is the cross power spectrum of the output and input signals, $G_{x x}(f)$ is the auto power spectrum of the input signal, and $f$ is the variable frequency. For each degree of freedom, the test procedure was repeated ten times and the average of FRFs was finally stored as the input-output transfer function, $\gamma$, given by Eq. (2):

$$
\gamma=G_{y x}(f) G_{x y}(f) /\left[G_{x x}(f) G_{y y}(f)\right]
$$

Where $G_{y y}(f)$ is the auto power spectrum of the output signal. The coherence function is a measure of consistency of the obtained data; being 1.0 for perfect consistency and 0 for no consistency. For all the data collected, almost perfect coherence values were recorded in the frequency range of interest.

\subsection{Test specimen}

The modal test was performed on a steel tank to evaluate its modal properties. The rectangular wall of the tank had the following geometric and material properties: height, $1000 \mathrm{~mm}$; width, $500 \mathrm{~mm}$; thickness, $8 \mathrm{~mm}$; Young`s modulus, $E_{s t}=2.05 \times 10^{5} \mathrm{~N} / \mathrm{mm}^{2}$; Poisson's ratio, 0.3 ; and mass density, $\rho_{s t}=7.7008 \times 10^{-5} \mathrm{~N} / \mathrm{mm}^{3}$. Fig. 1 shows the test setup and design details of the test specimen. As can be seen in this figure, the connections between two adjacent walls are such that little lateral resistance is induced by the side walls on the main wall. This will allow the cantilever behavior of the main wall, which is required for consistency with the analytical model.

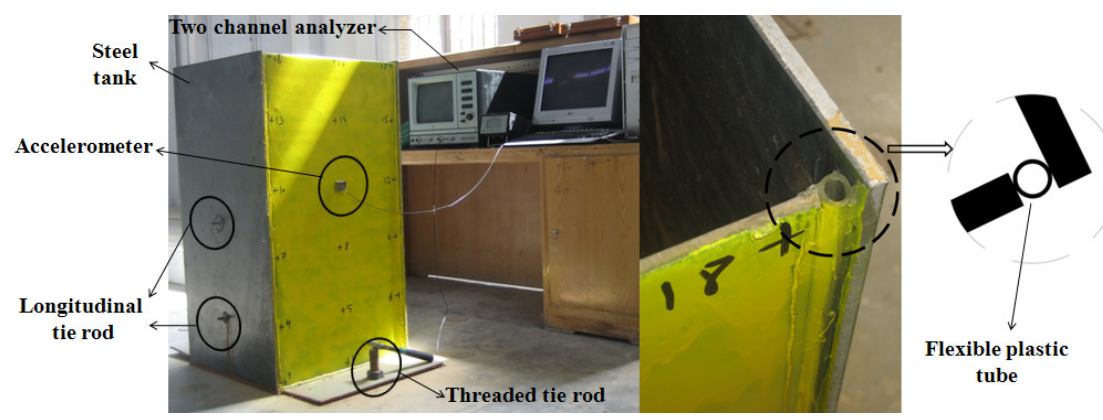

Figure 1: Detail of the test specimen. 


\subsection{Dynamic test set-up and results}

The dynamic response of the rectangular wall was recorded using one accelerometer placed at the degree of freedom No. 8. The impact excitation forces were applied at different degrees of freedom as shown in Fig. 2. The numbers of these degrees of freedom are chosen so that a smooth curve for the mode shapes can be derived from the experiment.

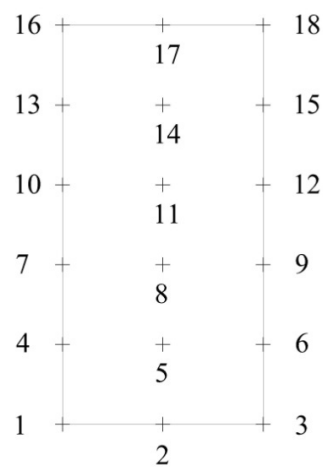

Figure 2: $\quad$ Degrees of freedom for dynamic test.

The tank was tested in two stages; empty and full (with $50 \mathrm{~mm}$ free board). A typical coherence function and a frequency response function for the empty tank is shown in Fig. 3.

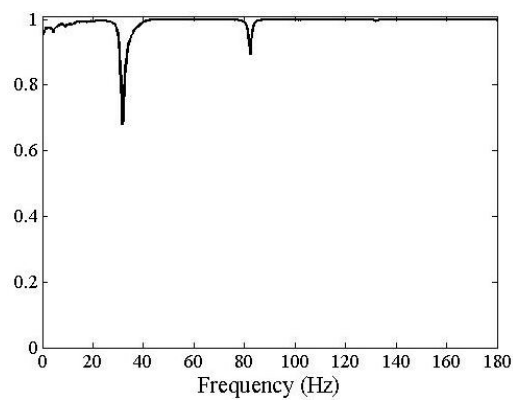

(a)

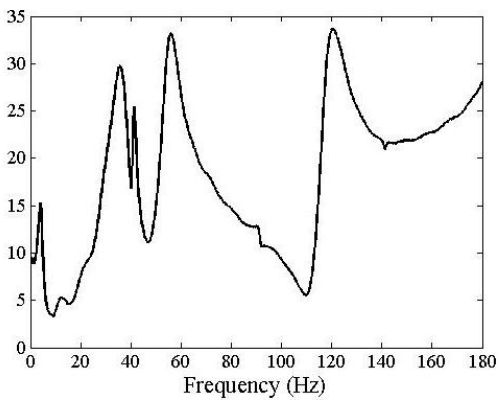

(b)

Figure 3: (a) Coherence function and (b) FRF function for empty tank.

Table 1 summarizes the results obtained from the experimental modal analysis for the first three modes. These modes are well defined and easily identifiable. Table 1 shows that as fluid height increases, the natural frequency of the coupled system decreases. 
Table 1: $\quad$ Frequency for the first three modes of the tank wall.

\begin{tabular}{lll}
\hline Frequency (Hz.) & Empty & Full \\
\hline$f_{1}$ & 6.75 & 3.10 \\
$f_{2}$ & 43.75 & 21.67 \\
$f_{3}$ & 122.6 & 71.78 \\
\hline
\end{tabular}

Fig. 4 shows the mode shapes of the tank wall corresponding to the first three natural frequencies of the empty tank.

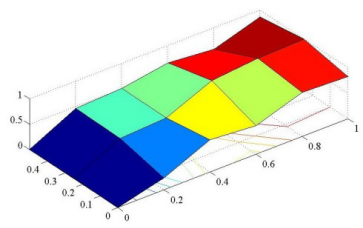

(a)

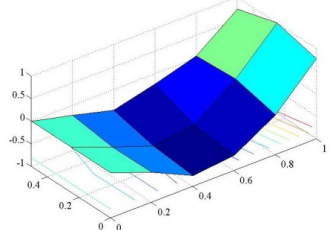

(b)

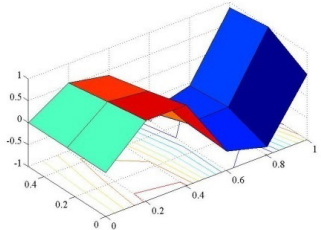

(c)

Figure 4: (a) First, (b) second and (c) third mode shapes of the wall obtained for the empty tank.

\section{Finite element analysis and results}

Two elements are utilized to analyze the system by Finite Element Method (FEM):

1- Bending beam elements with 2 nodes and 4 degrees of freedom in both displacement and rotation.

2- Fluid elements with 4 nodes and 4 pressure degrees of freedom.

These elements are utilized for modeling the structure and the fluid region, respectively. Typical finite element model is shown in Fig. 5. The numerical analyses are carried out in the frequency domain.

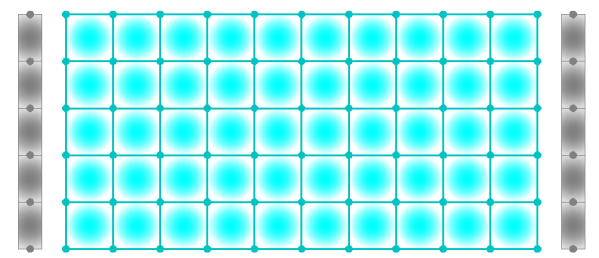

Figure 5: A typical finite element model for a rectangular tank. 
The coupled equation of the system can be given by (Aftabi Sana and Lotfi [9]):

$$
\left[\begin{array}{c:c}
\mathbf{K}-\omega^{2} \mathbf{M} & -\mathbf{B}^{\mathrm{T}} \\
\hdashline-\rho_{\mathrm{F}} \omega^{2} \mathbf{B} & \mathbf{H}-\left(\omega^{2} / c^{2}\right) \mathbf{G}
\end{array}\right]\left(\begin{array}{l}
\mathbf{D} \\
\hdashline \\
\mathbf{P}
\end{array}\right)=\left(\begin{array}{c}
-\mathbf{M} \mathbf{J} \mathbf{a}_{\mathrm{g}} \\
\hdashline-\rho_{\mathrm{F}} \mathbf{B} \mathbf{J} \mathbf{a}_{\mathrm{g}}
\end{array}\right)
$$

where $\mathbf{M}$ and $\mathbf{K}$ are the mass and stiffness matrices of the structure, $\mathbf{G}$ and $\mathbf{H}$ are the characteristic matrices of the fluid domain and $\mathbf{B}$ is the interaction matrix. These matrices are obtained by assembling the elemental matrices which are as follows for the structure:

$$
\begin{gathered}
\mathbf{M}_{e}=\frac{\rho \mathrm{L}}{420}\left[\begin{array}{c:c:c:c}
156 & 22 \mathrm{~L} & 54 & -13 \mathrm{~L} \\
\hdashline 22 \mathrm{~L} & 4 \mathrm{~L}^{2} & 13 \mathrm{~L} & -3 \mathrm{~L}^{2} \\
\hdashline 54 & 13 \mathrm{~L} & 156 & -22 \mathrm{~L} \\
\hdashline-13 \mathrm{~L} & -3 \mathrm{~L}^{2} & -22 \mathrm{~L} & 4 \mathrm{~L}^{2}
\end{array}\right], \\
\mathbf{K}_{e}=\frac{\mathrm{EI}}{\mathrm{L}^{3}}\left[\begin{array}{c:c:c:c}
12 & 6 \mathrm{~L} & -12 & 6 \mathrm{~L} \\
\hdashline 6 \mathrm{~L} & 4 \mathrm{~L}^{2} & -6 \mathrm{~L} & 2 \mathrm{~L}^{2} \\
\hdashline-12 & -6 \mathrm{~L} & 12 & -6 \mathrm{~L} \\
\hdashline 6 \mathrm{~L} & 2 \mathrm{~L}^{2} & -6 \mathrm{~L} & 4 \mathrm{~L}^{2}
\end{array}\right]
\end{gathered}
$$

The elemental matrices for the fluid domain are:

$$
\begin{aligned}
& \mathbf{G}_{e}=\mathrm{L}^{2}\left[\begin{array}{c:c:c:c}
\frac{1}{9} & \frac{1}{18} & \frac{1}{36} & \frac{1}{18} \\
\hdashline \frac{1}{18} & \frac{1}{9} & \frac{1}{18} & \frac{1}{36} \\
\hdashline \frac{1}{36} & \frac{1}{18} & \frac{1}{9} & \frac{1}{18} \\
\hdashline \frac{1}{18} & \frac{1}{36} & \frac{1}{18} & \frac{1}{9}
\end{array}\right], \\
& \mathbf{H}_{e}=\left[\begin{array}{c:c:c:c}
\frac{2}{3} & -\frac{1}{6} & -\frac{1}{3} & -\frac{1}{6} \\
\hdashline-\frac{1}{6} & \frac{2}{3} & -\frac{1}{6} & -\frac{1}{3} \\
\hdashline-\frac{1}{3} & -\frac{1}{6} & \frac{2}{3} & -\frac{1}{6} \\
\hdashline-\frac{1}{6} & -\frac{1}{3} & -\frac{1}{6} & \frac{2}{3}
\end{array}\right]
\end{aligned}
$$


and the elemental interaction matrix is as follows:

$$
\mathbf{B}_{e}=\left[\begin{array}{c:c:c:c}
\frac{7 \mathrm{~L}}{20} & \frac{\mathrm{L}^{2}}{20} & \frac{3 \mathrm{~L}}{20} & -\frac{\mathrm{L}^{2}}{30} \\
\hdashline \frac{3 \mathrm{~L}}{20} & \frac{\mathrm{L}^{2}}{30} & \frac{7 \mathrm{~L}}{20} & -\frac{\mathrm{L}^{2}}{20}
\end{array}\right] .
$$

Also, $\mathbf{J}$ is the matrix of rigid body motion, $\mathbf{a}_{\mathrm{g}}$ is the vector of ground accelerations, $\mathbf{D}$ is the vector of unknown nodal displacements of the structure, $\mathbf{P}$ is the vector of fluid dynamic pressure, $\omega$ is the exciting frequency, $c$ is the acoustic speed in the fluid and $\rho_{\mathrm{F}}$ is the fluid mass density.

It is noticeable that in Eq. (3), the material damping in the fluid and structure are negligible. By solving the linear system (Eq. 3), the nodal displacements of the wall and the nodal pressure of the fluid domain are attained. These results can be directly used for comparison with those of the proposed method.

The finite element method was implemented to obtain the dynamic response of the system to the unit ground motion. The finite element model is employed for the dynamic analysis of the proposed tank (Fig. 2). In the following, both empty and full tanks are investigated, simultaneously. Fig. 6 illustrates the free end displacement of the right wall for these two cases.

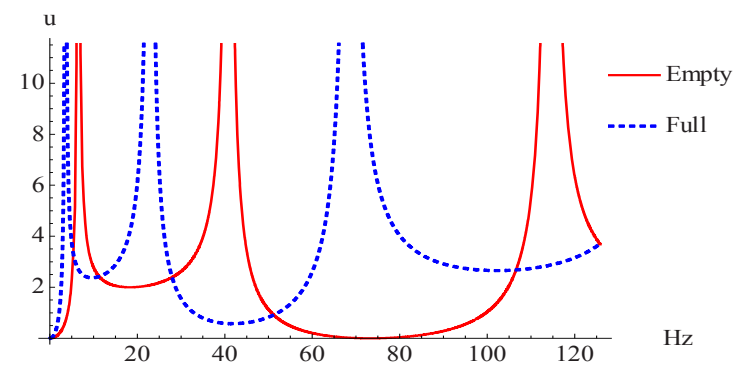

Figure 6: Comparison of the FRF for the empty and full tank.

It is obvious that when the tank is full, the mass of the system increases and the natural frequencies decreases. The computed natural frequencies for different modes are presented in Table 2. It is observed that the FEM results are in good agreement with experimental results.

Table 2: $\quad$ Frequency for the first three modes of the tank obtained from the experiment and FEM $(\mathrm{Hz})$.

\begin{tabular}{|c|c|c|c|c|c|c|}
\hline \multirow{2}{*}{ Mode } & \multicolumn{3}{|c|}{ Empty tank } & \multicolumn{3}{c|}{ Full tank } \\
\cline { 2 - 7 } & Experimental & FEM & Difference (\%) & Experimental & FEM & Difference (\%) \\
\hline 1 & 6.57 & 6.52 & 0.7 & 3.1 & 3.57 & 13 \\
\hline 2 & 43.75 & 40.87 & 6.58 & 21.67 & 22.92 & 5.4 \\
\hline 3 & 122.6 & 114.46 & 6.63 & 71.78 & 68.53 & 4.52 \\
\hline
\end{tabular}


The other case is the full tank with flexible walls and large rigidity value (3EI). Fig. 7 shows the effect of wall rigidity on the FRF for the displacement of the structure and the dynamic pressure of the fluid. As expected, the natural frequencies are amplified as the wall rigidity increases.

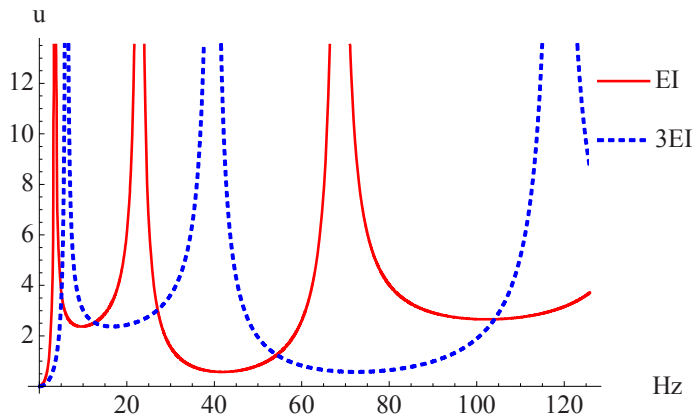

Figure 7: Effect of EI (flexibility of the right wall) on the FRF.

The pressure distributions obtained from finite element method are presented for the fluid-structure system. Fig. 8 shows the pressure contours plotted for some exciting frequencies.

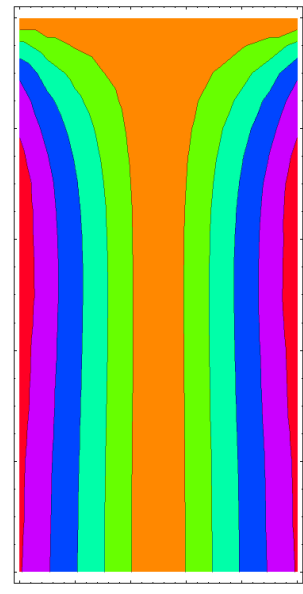

$\omega=1.6 \mathrm{~Hz}$

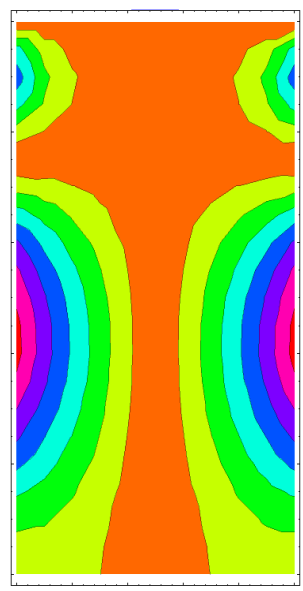

$\omega=25 \mathrm{~Hz}$

Figure 8: The contours of the fluid dynamic pressure.

\section{Conclusions}

The free vibration of liquid storage tanks was studied in this paper. The study included both experimental and numerical investigations. In the experimental part of the study, a steel tank was manufactured and the modal test was 
performed on the specimen to evaluate its modal properties. In addition, a finite element analysis has been carried out. The numerical results from this analysis were compared with the experimental values. The comparison shows that the finite element results are generally in good agreement with the experimental values. In addition, effect of wall flexibility on the dynamic response of system is investigated. The results show that the wall flexibility has major effects on seismic behavior of liquid tanks and should be considered in design criteria of tanks.

\section{References}

[1] Rebouillat S., Liksonov D., Fluid-structure interaction in partially filled liquid containers: A comparative review of numerical approaches, Computers \& Fluids 39, 739-746, 2010.

[2] Ghaemmaghami A.R., Kianoush M.R., Effect of wall flexibility on dynamic response of concrete rectangular liquid storage tanks under horizontal and vertical ground motions, Journal of Structural Engineering (ASCE), 136, 441-451, 2010.

[3] Kim J., Koh H., Kwah I., Dynamic response of rectangular flexible fluid containers, Journal of Engineering Mechanics (ASCE), 122, 807-816, 1996.

[4] Koh H.M., Kim J., Park J.H., Fluid-structure interaction analysis of 3-D rectangular tanks by a vibrationally coupled BEM-FEM and comparison with test results, Earthquake Engineering and Structural Dynamics, 27, 109124, 1998.

[5] Park, J. H., Koh, H. M. and Kim, J. Liquid-structure interaction analysis by coupled boundary element-finite element method in time domain. Proceedings of the 7th International Conference on Boundary Element Technology, BE-TECH/92, Computational Mechanics Publication, Southampton, England. 89-92, 1992.

[6] Chen JZ, Kianoush MR. Seismic response of concrete rectangular tanks for liquid containing structures, Can J Civil Eng, 32:739 52, 2005.

[7] Virella JC, Godoy LA, Suarez LE. Fundamental modes of tank-liquid under horizontal motions. Engineering Structure, 28, 1450-1461, 2006.

[8] Biswal KC, Bhattacharyya SK, Sinha PK. Dynamic response analysis of a liquid-filled cylindrical tank with annual baffle, J. Sound and Vibration, 274(1-2), 13-37, 2004.

[9] Aftabi Sani A., Lotfi V., Linear dynamic analysis of arch dams utilizing modified efficient fluid hyper-element, Engineering Structures, 29, 26542661, 2007. 